\title{
Chance-Constrained Economic Dispatch with Renewable Energy and Storage
}

\author{
Jianqiang Cheng • Richard Li-Yang Chen • \\ Habib N. Najm • Ali Pinar • Cosmin \\ Safta · Jean-Paul Watson
}

Received: date / Accepted: date

\begin{abstract}
Increasing penetration levels of renewables have transformed how power systems are operated. High levels of uncertainty in production make it increasingly difficulty to guarantee operational feasibility; instead, constraints may only be satisfied with high probability. We present a chance-constrained economic dispatch model that efficiently integrates energy storage and high renewable penetration to satisfy renewable portfolio requirements. Specifically, we require that wind energy contribute at least a prespecified proportion of the total demand and that the scheduled wind energy is deliverable with high probability. We develop an approximate partial sample average approximation (PSAA) framework to enable efficient solution of large-scale chance-constrained economic dispatch problems. Computational experiments on the IEEE-24 bus system show that the proposed PSAA approach is more accurate, closer to the prescribed satisfaction tolerance, and approximately 100 times faster than standard sample average approximation. Finally, the improved efficiency of our PSAA approach enables solution of a larger WECC-240 test system in minutes.
\end{abstract}

This work was funded by the Laboratory Directed Research and Development (LDRD) program of the Sandia National Laboratories. Sandia National Laboratories is a multimission laboratory managed and operated by National Technology \& Engineering Solutions of Sandia, LLC, a wholly owned subsidiary of Honeywell International Inc., for the U.S. Department of Energys National Nuclear Security Administration under contract DE-NA0003525. The views expressed in the article do not necessarily represent the views of the U.S. Department of Energy or the United States Government. This research has also been supported in part by the Bisgrove Scholars program (sponsored by Science Foundation Arizona).

Jianqiang Cheng

Department of Systems and Industrial Engineering, University of Arizona, Tucson, AZ 85721, USA.

E-mail: jqcheng@email.arizona.edu

Richard Li-Yang Chen · Habib N. Najm · Ali Pinar · Cosmin Safta

Sandia National Laboratories, Livermore, CA 94551, USA

E-mail: \{rlchen, hnnajm, apinar, csafta\}@sandia.gov

Jean-Paul Watson

Sandia National Laboratories, Albuquerque, NM 87185, USA

E-mail: jwatson@sandia.gov 
Keywords Chance constraints - Sample average approximation · Partial sample average approximation · Economic dispatch · Renewable energy integration . Energy storage

Mathematics Subject Classification (2000) MSC 90C15 - MSC 90C59 • MSC 90C90

\section{Introduction}

High penetration levels of renewable generation are transitioning from goals to requirements. For example, California's Renewable Portfolio Standard (RPS) requires that renewable generation sources comprise $33 \%$ of energy supply by 2020 [3]; other system operators are facing similar mandates. Given these aggressive targets, renewable energy integration will pose significant challenges for power system reliability, due to the intermittency and limited predictability of renewable energy production [34]. To address these challenges, advanced operations models and corresponding solution methods are required to ensure system reliability and cost-effectiveness.

The unit commitment (UC) problem, which deals with scheduling power generation to meet anticipated demand, is a fundamental problem in power systems operations. Economic dispatch (ED), closely related to UC, minimizes production costs while satisfying demand and transmission constraints given a fixed set of committed, active thermal generators; in UC, the commitments are additionally determined. UC and ED are traditionally formulated as deterministic problems, but the high levels of uncertainty that are inherent in renewable energy production require alternate formulations that effectively and directly account for such uncertainty, avoiding strict reliance on deterministic reserve margins. For more detailed information about UC and ED, we refer the reader to [22] and [38].

Approaches to handle uncertainty in UC and ED can be grouped into two categories: robust optimization $([1,16,18])$ and stochastic programming $([26,37,39])$. Robust optimization does not rely on explicit characterizations of probability distributions of random variables. Rather, the focus is on the ranges of these variables. The goal of robust optimization is to provide solutions that are feasible given any realization of the random variables, subject to the specified interval bounds. Thus, commitment and dispatch decisions are made considering the worst-case of a specified uncertainty set. Construction of uncertainty sets and the connection between interval bounds and cost have drawn recent attention $[1,18]$.

In contrast to robust optimization, stochastic programming approaches assume probability distributions of random variables are known or can be accurately estimated. Stochastic programming variants of UC are typically formulated as two-stage decision models and solved via decomposition methods such as Benders' decomposition [35] and progressive hedging [10,27]. An important variant of stochastic programming is chance-constrained programming, which requires that constraints involving uncertain parameters be satisfied with high probability. Wang et al. [37] consider a chance constraint at the first decision stage of UC to ensure that, with high probability, a large proportion of generated wind power was utilized. Chance constraints were also integrated into stochastic ED by [26] and [39]. Wu et al. [39] proposed a day-ahead model where reserve requirements and 
line capacity constraints are satisfied with a high probability, while Qiu and Wang [26] introduced a chance-constrained model that ensured, with high probability, that the total amount of wind energy utilized was at least a specified fraction of the total wind energy available.

Because chance-constrained programs are among the most computationally challenging optimization problems [20], especially jointly chance-constrained variants, practical tractability of jointly chance-constrained ED problems is currently restricted to small-scale systems. The computational challenge is further compounded if unit commitment decisions are integrated within the dispatch problem, resulting in a chance-constrained mixed-integer optimization problem. For example, leveraging state-of-the-art decomposition algorithms, Wang et al. [37] managed to solve a chance-constrained UC/ED problem for a modified 118-bus system, but with only a single wind site. For practical-scale power systems with multiple wind sites, the solution of chance-constrained UC/ED within practical time limits is currently infeasible. As the ED problem is an integral component of combined UC/ED problem, we focus our attention on first achieving tractability for the chance-constrained ED problem, where unit commitment decisions are fixed. Additionally, our proposed approach is directly applicable to other forms of ED problems, such as security-constrained ED.

We consider a novel chance-constrained economic dispatch model, which efficiently integrates a large amount of renewable resources (e.g., wind) and energy storage to ensure that a significant amount of the total electricity demand can be satisfied by renewable output with a prescribed (high) probability. Energy storage can help mitigate the effects of renewable energy variability and uncertainty by storing excess wind energy for use in subsequent periods, and consequently has drawn significant interest from both industry and academia in recent years $([6,13$, $33]$ ). For more information on energy storage, we refer the reader to recent surveys $[5,31]$.

In our formulation, we require that a significant portion of the total demand must be met with wind turbine output. The difference between our chance constraints and those in [26] is that we require the scheduled wind power delivery to be at least $\beta$ percent of total demand and achievable with high probability $1-\alpha$, which is congruent with states' RPS mandates. This is in contrast to a chance constraint on total wind production, which is irrespective of actual demand. We follow $[16,26,37]$ and assume that wind output can be curtailed if the amount of wind generation delivered (cleared) does not match the actual wind generation output.

While chance constraints present a natural language to model operational problems under uncertainty, they come at the cost of increased computational complexity. There are two main challenges. First, feasible sets of chance-constrained problems are generally not convex. Therefore, it is difficult to find a globally optimal solution. Second, even for a convex problem, it is difficult to check feasibility of a candidate solution as this generally requires multi-dimensional integration. Nemirovski and Shapiro [20] note that evaluating the distribution of a weighted sum of uniformly distributed independent random variables is NP-hard. Consequently, most research applies approximation techniques to achieve near-optimal solutions. One approach is to employ convex (or tractable) approximations (see, e.g., [20]). Other approaches use sample-based techniques, such as sample average approximation (SAA) [19,23]. The basic idea of SAA is to approximate the true 
distribution of random variables by empirical distributions estimated from Monte Carlo (MC) samples. SAA can provide high-quality solutions when the number of scenarios is sufficiently large. Due to the convergence rate of MC methods, the required number of samples increases quadratically with required accuracy. Unfortunately, "big-M" reformulations $([19,32])$ of SAA problems introduce a number of binary decision variables equal to the number of scenarios, which typically yields computationally difficult optimization models. However, under some conditions on the underlying uncertainty, a modified SAA, known as partial sample average approximation (PSAA) [9], can be leveraged to solve chance-constrained problems without introducing binary variables. In this paper, we present a PSAA approach to the ED problem with uncertain generation and energy storage, compare our results to SAA, and show that PSAA can enable solution of larger instances in a fraction of the time and with improved accuracy.

The contributions of this paper are summarized as follows:

1. We introduce a novel chance-constrained economic dispatch model with energy storage. With the new model, users can explicitly control the ratio of renewable energy's contribution to the total demand (production), which is consistent with state renewable portfolio standards and goals [28].

2. We present an approximate PSAA approach to effectively solve the chanceconstrained ED model and demonstrate its computational efficiency. Since an exact PSAA reformulation yields a non-linear program, we apply piecewise approximation schemes to derive a linear program. This sharply contrasts to the SAA approach, which results in a mixed-integer linear program. The proposed approximate PSAA approach is generally applicable to other forms of chance-constrained ED models, e.g., as in [26].

\section{Preliminaries}

\subsection{Chance-Constrained Optimization Models}

A general chance-constrained model is defined as follows:

$$
\begin{array}{rl}
\min _{x} & f(x) \\
\text { s.t. } & \mathbb{P}\left\{g_{j}(x, \xi) \geq 0, \quad j=1, \ldots, m\right\} \geq 1-\alpha ; \quad x \in X
\end{array}
$$

where $\xi$ denotes a random vector, $\mathbb{P}\{E\}$ denotes the probability that the constraints $E$ are satisfied, and the set $X$ represents additional deterministic constraints. The input parameter $\alpha \in[0,1]$ is a parameter chosen by decision makers, and represents the tolerance for risk, or more explicitly, the acceptable probability for violating the chance constraints. When $m>1$, constraint (1) is known as a joint chance constraint; otherwise, it is known as an individual chance constraint.

Chance-constrained optimization was introduced by Charnes et al. [7], and has subsequently attracted attention due to its wide applicability in engineering, telecommunications, and finance. For a review of the theory and applications of chance-constrained optimization, we refer to $[24,30]$. It is widely known that chance-constrained optimization models are generally difficult to solve, even at small scale. Consequently, various approximation methods have been introduced. Among these approaches, we next present four methods: Bonferroni approximation [8,20], Scenario approach (SA) [4], SAA, and PSAA. 


\subsection{Bonferroni Approximation}

A popular approximation for jointly chance-constrained problems is based on Bonferronis inequality, which decomposes the joint chance constraint into $m$ individual chance constraints. When $\sum_{j=1}^{m} \alpha_{j}=\alpha$, we have

$\mathbb{P}\left\{g_{j}(x, \xi) \geq 0\right\} \geq 1-\alpha_{j}, \quad j=1, \ldots, m \Rightarrow \mathbb{P}\left\{g_{j}(x, \xi) \geq 0, \quad j=1, \ldots, m\right\} \geq 1-\alpha$.

Accordingly, one can obtain a conservative approximation of problem (1):

$$
\begin{array}{ll}
\min _{x} & f(x) \\
\text { s.t. } & \mathbb{P}\left\{g_{j}(x, \xi) \geq 0\right\} \geq 1-\alpha_{j}, \quad j=1, \ldots, m ; \quad x \in X .
\end{array}
$$

Further, if $g_{j}(x, \xi)$ is separable, i.e., $g_{j}(x, \xi)=g_{j}(x)-h_{j}(\xi)$, then we have the following deterministic reformulation of the Bonferroni approximation problem

$$
\begin{array}{ll}
\min _{x} & f(x) \\
\text { s.t. } & g_{j}(x) \geq \Phi_{j}^{-1}\left(1-\alpha_{j}\right), \quad j=1, \ldots, m ; \quad x \in X
\end{array}
$$

where $\Phi_{j}^{-1}(\cdot)$ is the inverse of the cumulative distribution function of $h_{j}(\xi)$.

Remark 2.21 In our subsequent numerical tests, we set $\alpha_{j}=\frac{\alpha}{m}, j=1, \ldots, m$.

\subsection{Scenario Approach (SA)}

Under the assumption that we can sample from the distribution of $\xi$, then a scenario approximation [4] of problem (1) is

$$
\begin{array}{ll}
\min _{x} & f(x) \\
\text { s.t. } & g\left(x, \hat{\xi}^{k}\right) \geq 0, k=1, \ldots, N ; x \in X
\end{array}
$$

where $g(x, \xi)=\min _{j=1}^{m} g_{j}(x, \xi)$, and scenarios $\hat{\xi}^{1}, \ldots, \hat{\xi}^{N}$ are independent samples drawn from the distribution of $\xi$. One advantage of this approach is that when $X$ is a convex set and $g(x, \xi)$ is concave in $\mathrm{x}$ for each $\xi$, the scenario approximation is a convex optimization problem. However, the approach can be too conservative [19], as it requires that all sampled constraint sets be satisfied.

\subsection{Sample Average Approximation (SAA)}

The idea of SAA is to approximate the expectation of a random variable by its sample mean. We begin by formulating the general chance-constrained optimization model as an expectation

$$
\mathbb{P}\{g(x, \xi) \geq 0\}=\mathbb{E}[\mathbb{I}(g(x, \xi)]
$$

where $\mathbb{I}(\cdot)$ is 1 if $\cdot \geq 0$ and 0 otherwise. We then approximate the expectation via a sample mean, i.e., $\frac{1}{N} \sum_{t=1}^{N} \mathbb{I}\left(g\left(x, \hat{\xi}^{t}\right)\right)$ where scenarios $\hat{\xi}^{1}, \ldots, \hat{\xi}^{N}$ are independent 
samples drawn from the distribution of $\xi$. The SAA formulation of problem (1) can then be written as:

$$
\begin{array}{ll}
\min _{x} & f(x) \\
\text { s.t. } & \frac{1}{N} \sum_{k=1}^{N} \mathbb{I}\left(g\left(x, \hat{\xi}^{k}\right)\right) \geq 1-\alpha ; \quad x \in X .
\end{array}
$$

Problem (3) can be reformulated as

$$
\begin{array}{cl}
\min & f(x) \\
\text { s.t. } & g\left(x, \hat{\xi}^{k}\right)+M y_{k} \geq 0, k=1, \ldots, N \\
& \sum_{k=1}^{N} y_{k} \leq N \alpha \\
& y_{k} \in\{0,1\}, k=1, \ldots, N ; \quad x \in X
\end{array}
$$

where the binary indicator variable $y_{k}$ denotes whether constraints $g\left(x, \hat{\xi}^{k}\right)$ are satisfied and $M$ denotes a sufficiently large constant such that $g\left(x, \hat{\xi}^{k}\right)+M \geq 0$ is satisfied for all $x \in X$ and $k \in\{1, \ldots, N\}$. For additional details on SAA approaches for solving chance-constrained optimization models, we refer to [19, 23].

\subsection{Partial Sample Average Approximation (PSAA)}

SAA can in theory yield accurate solutions when the number of scenarios is sufficiently large [19]. However, because the number of binary variables $y_{k}$ is equal to the number of scenarios, solving an SAA model (4) can pose a significant computational challenge. Recently, Cheng et al. [9] introduced PSAA, which only samples a portion of random variables, in contrast to SAA which samples all of the random variables. The PSAA approach makes use of the independence of some of the random variables for stepwise evaluation of the expectation. Specifically, $\mathbb{E}_{\left(\xi_{1}, \xi_{2}\right)} g\left(\xi_{1}, \xi_{2}\right)=\mathbb{E}_{\xi_{1}} \mathbb{E}_{\xi_{2}} g\left(\xi_{1}, \xi_{2}\right)$ when the random vectors $\xi_{1}$ and $\xi_{2}$ are independent. PSAA then applies SAA to approximate the inner (or outer) expectation.

PSAA assumes that $\xi=\left(\xi_{1}, \xi_{2}\right)$ such that $\xi_{1}$ is independent of $\xi_{2}$. The probability of satisfying the joint constraints is then formulated as an expectation: $\mathbb{P}\{g(x, \xi) \geq 0\}=\mathbb{E}[\mathbb{I}(g(x, \xi))]=\mathbb{E}_{\xi_{1}, \xi_{2}}[\mathbb{I}(g(x, \xi))]$. As $\xi_{1}$ and $\xi_{2}$ are independent, the expectation can be calculated as $\mathbb{E}_{\xi_{1}, \xi_{2}}\left[\mathbb{I}\left(g\left(x, \xi_{1}, \xi_{2}\right)\right)\right]=\mathbb{E}_{\xi_{1}} \mathbb{E}_{\xi_{2}}\left[\mathbb{I}\left(g\left(x, \xi_{1}, \xi_{2}\right)\right)\right]$ where PSAA now replaces one of the two expectations by its sample mean. For instance, if we replace the inner expectation by a sample mean (where $\hat{\xi}_{2}{ }^{1}, \ldots, \hat{\xi}_{2}{ }^{N}$ are $N$ independent samples of $\xi_{2}$ ), then the PSAA formulation of (1) is as follows:

$$
\begin{aligned}
& \min _{x} f(x) \\
& \frac{1}{N} \sum_{k=1}^{N} \mathbb{E}_{\xi_{2}}\left[\mathbb{I}\left(g\left(x, \xi_{1}, \hat{\xi}_{2}^{k}\right)\right)\right] \geq 1-\alpha ; \quad x \in X
\end{aligned}
$$


Problem (5) is equivalent to the following optimization problem.

$$
\begin{aligned}
& \min _{x} f(x) \\
& \frac{1}{N} \sum_{k=1}^{N} \mathbb{P}\left\{g\left(x, \xi_{1}, \hat{\xi}_{2}^{k}\right) \geq 0\right\} \geq 1-\alpha ; \quad x \in X
\end{aligned}
$$

Problem (6) is equivalent to the following optimization problem.

$$
\begin{aligned}
& \min _{x} f(x) \\
& \mathbb{P}\left\{g\left(x, \xi_{1}, \hat{\xi}_{2}^{k}\right) \geq 0\right\} \geq y_{k}, k=1, \ldots, N, \\
& \quad \frac{\sum_{k=1}^{N} y_{k}}{N} \geq 1-\alpha, \\
& y_{k} \geq 0, k=1, \ldots, N, \quad x \in X
\end{aligned}
$$

PSAA retains the general idea of SAA, in that the expectation is replaced by a sample mean. Compared with problem (4) (SAA reformulation), problem (7) (PSAA reformulation) has only continuous variable $y_{k}$. However, there are $N$ new chance constraints. We demonstrate below that Eq. (7) has a convex approximation for certain problem classes. For example, when $g\left(x, \xi_{1}, \xi_{2}\right)=a(x)^{T} \xi_{2}-\xi_{1}$ where $a(x)$ is an affine vector of $x$, Eq. (7) becomes

$$
\mathbb{P}\left\{a(x)^{T} \xi_{2}^{k} \geq \xi_{1}\right\} \geq y_{k} \Leftrightarrow a(x)^{T} \hat{\xi}_{2}^{k} \geq \Phi^{-1}\left(y_{k}\right)
$$

where $\Phi^{-1}(p)$ is the inverse cumulative distribution function of $\xi_{1}$. Further, if we can find a convex approximation for function $\Phi^{-1}(p)$, then Eq. (7) has a convex approximation. For additional details we refer the reader to [9].

Remark 2.51 As it is easier to approximate the inverse cumulative distribution function of $\xi_{1}$ when $\xi_{1}$ is univariate, PSAA approximation usually chooses only one random variable to not be sampled in order to facilitate finding a tractable approximation for Eq. (7).

\section{Mathematical Formulation}

We first introduce the core sets, parameters, and decision variables of the model.

\section{Nomenclature}

\section{Sets and Indices}

$\mathcal{I} \quad$ Set of buses. Indexed by $i$ for individual buses, $i$ and $j$ for pairs of buses.

$\mathcal{I}_{r} \quad$ Set of buses with renewable generators.

$I \quad$ Number of buses. $I=|\mathcal{I}|$.

$I_{r} \quad$ Number of buses with renewable generators. $I_{r}=\left|\mathcal{I}_{r}\right|$.

$\mathcal{G} \quad$ Set of generation units. Indexed by $g$.

$G \quad$ Number of generation units. $G=|\mathcal{G}|$.

$\mathcal{G}_{i} \quad$ Set of generation units located at bus $i \in \mathcal{I}$. 
$\mathcal{E} \quad$ Set of directed transmission lines connecting pairs of buses. Indexed by $e=(i, j)$.

$E \quad$ Number of directed transmission lines. $E=|\mathcal{E}|$.

$\mathcal{E}_{i}^{+} \quad$ Set of transmission lines oriented into bus $i \in \mathcal{I}$.

$\mathcal{E}_{i}^{-} \quad$ Set of transmission lines oriented out of bus $i \in \mathcal{I}$.

$\mathcal{T}$ Set of time periods in planning horizon. Indexed by $t$.

$T \quad$ Number of time periods. $T=|\mathcal{T}|$.

\section{Parameters}

$B_{e} \quad$ Susceptance of transmission line $e$.

$F_{e} \quad$ Power flow limit of transmission line $e$.

$D_{i}^{t} \quad$ Demand (load) at bus $i$ in time period $t$.

$P_{g}^{\min } \quad$ Lower limit on power output for generation unit $g$.

$P_{g}^{\max } \quad$ Upper limit on power output for generation unit $g$.

$R_{g}^{d} \quad$ Maximum ramp-down rate for generation unit $g$ between adjacent time periods.

$R_{g}^{u} \quad$ Maximum ramp-up rate for generation unit $g$ between adjacent time periods.

$R_{s}^{d}(i) \quad$ Maximum storage discharge at bus $i$ in a time period.

$R_{s}^{u}(i) \quad$ Maximum storage charge at bus $i$ in a time period.

$Q_{i}^{\min } \quad$ Lower limit on capacity for storage unit at bus $i$.

$Q_{i}^{\max } \quad$ Upper limit on capacity for storage unit at bus $i$.

$C_{g}(\cdot) \quad$ Production cost function for generation unit $g$.

$\tilde{p}_{w_{i}}^{t} \quad$ Wind power available at bus $i$ in time period $t$.

\section{Decision Variables}

$p_{g}^{t} \quad$ Power delivered by generation unit $g$ in time period $t$.

$f_{e}^{t} \quad$ Power flow on transmission line $e$ in time period $t$.

$\theta_{i}^{t} \quad$ Voltage phase angle at bus $i$ in time period $t$.

$p \quad$ Length $G \times T$ power output vector.

$f$ Length $E \times T$ power flow vector.

$\boldsymbol{\theta}$ Length $I \times T$ voltage phase angle vector.

$p_{w_{i}}^{t} \quad$ Wind power production at bus $w_{i}$ in time period $t$.

$q_{i}^{t} \quad$ Energy storage level at bus $i$ in time period $t$.

Given a fixed unit commitment vector $\boldsymbol{x}$, the objective in the deterministic (multi-period) economic dispatch problem is to minimize total production cost while meeting demand, subject to constraints associated with unit ramping limitations and linearized (DC) approximations of AC power flow, as follows:

$$
\begin{array}{ll}
\min _{\boldsymbol{f}, \boldsymbol{p}, \boldsymbol{\theta}} & \sum_{g \in \mathcal{G}} \sum_{t \in \mathcal{T}} C_{g}\left(p_{g}^{t}\right) \\
\text { s.t. } & \sum_{g \in \mathcal{G}_{i}} p_{g}^{t}+\sum_{e \in \mathcal{E}(i)^{+}} f_{e}^{t}-\sum_{e \in \mathcal{E}(i)^{-}} f_{e}^{t}=D_{i}^{t}, \forall i \in \mathcal{I}, \forall t \in \mathcal{T} \\
& B_{e}\left(\theta_{i}^{t}-\theta_{j}^{t}\right)-f_{e}^{t}=0 \forall e=(i, j) \in \mathcal{E}, \forall t \in \mathcal{T} \\
& -F_{e} \leq f_{e}^{t} \leq F_{e} \quad \forall e \in \mathcal{E}, \forall t \in \mathcal{T} \\
& P_{g}^{\min } \leq p_{g}^{t} \leq P_{g}^{\max } \quad \forall g \in \mathcal{G}, \forall t \in \mathcal{T} \\
& -R_{g}^{d} \leq p_{g}^{t}-p_{g}^{t-1} \leq R_{g}^{u} \quad \forall g \in \mathcal{G}, \forall t \in \mathcal{T}
\end{array}
$$


The generation cost $C_{g}\left(p_{g}^{t}\right)$ is represented by a convex quadratic function. Constraints (10b)-(10f) require (in order): power balance at each bus; power flow on lines is proportional to the difference in voltage phase angles at the terminal buses; transmission line capacity limits are satisfied; lower and upper bounds for generation output levels are met; and generation ramp-up/ramp-down limits are satisfied.

Incorporating wind resources (and renewable resources in general) and energy storage devices requires some changes to this basic formulation. First, because wind generation is uncertain, the formulation must be stochastic. Consequently, for the wind resources at a bus $i$, the variable $p_{w_{i}}^{t}$ represents the amount of wind output scheduled for delivery in period $t$. Secondly, we need to take into account the amount of energy retained in storage devices. Here, we let $q_{i}^{t}$ denote the energy level of the storage unit at bus $i$ at time $t$. Leveraging these additional variables, our (multi-period) chance-constrained economic dispatch model is then given as follows:

$$
\begin{aligned}
\min _{\boldsymbol{f}, \boldsymbol{p}, \boldsymbol{\theta}, \boldsymbol{q}} & \sum_{g \in \mathcal{G}} \sum_{t \in \mathcal{T}} C_{g}\left(p_{g}^{t}\right) \\
\text { s.t. } & p_{w_{i}}^{t}+\sum_{g \in \mathcal{G}_{i}} p_{g}^{t}+\sum_{e \in \mathcal{E}(i)^{+}} f_{e}^{t}-\sum_{e \in \mathcal{E}(i)^{-}} f_{e}^{t}= \\
& D_{i}^{t}+q_{i}^{t}-q_{i}^{t-1}, \forall i \in \mathcal{I}_{r}, \forall t \in \mathcal{T} \\
& \sum_{g \in \mathcal{G}_{i}} p_{g}^{t}+\sum_{e \in \mathcal{E}_{. i}} f_{e}^{t}-\sum_{e \in \mathcal{E}_{i} .} f_{e}^{t}=D_{i}^{t}, \\
& \text { Constraints }(10 \mathrm{c})-(10 \mathrm{f}) \\
& -R_{s}^{d}(i) \leq q_{i}^{t}-q_{i}^{t-1} \leq R_{s}^{u}(i), \forall i \in \mathcal{I}_{r}, \forall t \in \mathcal{T}, \forall t \in \mathcal{T} \\
& Q_{i}^{\min } \leq q_{i}^{t} \leq Q_{i}^{\max }, t=1, \ldots T-1, \forall i \in \mathcal{I}_{r} \\
& \sum_{i \in \mathcal{I}_{r}} \sum_{t=1}^{T} p_{w_{i}}^{t} \geq \beta \sum_{i \in \mathcal{I}} \sum_{t=1}^{T} D_{i}^{t}, \\
& \mathbb{P}\left\{p_{w_{i}}^{t} \leq \tilde{p}_{w_{i}}^{t}, \forall t \in \mathcal{T}, \forall i \in \mathcal{I}_{r}\right\} \geq 1-\alpha
\end{aligned}
$$

In this formulation, we assume that storage devices are installed at the same buses as wind generators but this assumption can be easily relaxed without structurally changing the problem. The parameter $q_{i}^{0}, \forall i \in \mathcal{I}_{r}$, denotes the initial storage level at bus $i$. The standard power balance constraint (11a) is augmented with wind scheduled dispatch variables $p_{w_{i}}^{t}$ and nodal energy charge/discharge as given by the difference between storage levels at successive time periods $q_{i}^{t}-q_{i}^{t-1}$. Constraint (11c) ensures that charge and discharge ramp limits on storage devices are satisfied. Constraint (11d) imposes upper and lower bounds on the maximum and minimum storage levels. Note that storage levels must be at least $Q_{i}^{\text {min }}$ to prevent end of horizon behaviors in which batteries are completely discharged to minimize production cost. This simplified storage model follows [41] and can be easily augmented to include other storage constrains (e.g. loss of efficiency during discharge). Constraints (11e-11f) together enforce the RPS requirement that at 
least $\beta$ fraction of the total demand is met using wind power with probability at least $1-\alpha$. Specifically, Eq. (11e) dictates that wind delivered (cleared) must satisfy at least $\beta$ fraction of the total demand over the planning horizon. However, (11e) alone is not sufficient to guarantee that the amount of wind scheduled for delivery $p_{w_{i}}^{t}$ is feasible with high probability. Chance constraint (11f) ensures that the chosen wind schedule $p_{w_{i}}^{t} \forall t \in \mathcal{T}$ and $\forall i \in \mathcal{I}_{r}$ is jointly feasible with probability at least $1-\alpha$. In other words, the joint probability that the wind power scheduled for delivery, $p_{w_{i}}^{t} \forall t \in \mathcal{T}$ and $\forall i \in \mathcal{I}_{r}$, is less than or equal to the actual wind power production $\tilde{p}_{w_{i}}^{t}$ is at least $1-\alpha$.

Remark 3.01 For clarity, we present a simple model with a single storage device at each candidate bus. However, our model can be easily extended to multiple storage devices where capacities and charging/discharging profiles vary.

Remark 3.02 There are a number of practical considerations that must be addressed before formulation (11) can be viable in real-world power systems contexts. Specifically, issues will likely arise when the realized wind power $\tilde{p}_{w_{i}}^{t}$ is significantly different from the scheduled wind power $p_{w_{i}}^{t}$. We observe that (11a-11f) constrains the amount of power that can be injected into and withdrawn from storage devices and requires that a specific fraction of total demand must be met by wind. If realized wind power greatly exceeds storage device ramping limits and/or capacity or, alternatively, if the amount of realized wind power is smaller than the requisite mandate, then there is a high probability that the stochastic optimization problem (11) will be infeasible. In the case where realized wind power exceeds scheduled wind power, the additional power can be curtailed, as can and does occur in practice. Discrepancies between scheduled and realized wind power in both directions can be addressed by imposing an additional chance constraint on the satisfaction of (11c-11d) with high probability, or by imposing similar chance constraints in the long-term (wind and storage) capacity expansion problem to ensure that adequate wind and storage is added to address the uncertainty associated with wind power during operations.

Remark 3.03 The parameter $\beta$ in Eq. (11e) should be chosen dynamically, as the availability of wind power can vary significantly during different periods of the year.

Given $\beta^{*}$, a mandated RPS level, and satisfaction probability $\alpha$, system operators are faced with the problem of how to choose $\beta$ dynamically for day-to-day operations such that the required RPS is satisfied in the long term with high probability.

Theorem 31 Given an RPS mandate $\beta^{*}$, if $\beta$ is selected such that $\beta \geq \beta^{*} /(1-\alpha)$ and the resulting dispatch of wind power $\hat{p}_{w_{i}}^{t}=\min \left\{p_{w_{i}}^{t *}, \tilde{p}_{w_{i}}^{t}\right\}$ is feasible where $p_{w_{i}}^{t *}$ is components of an optimal solution of (11) with $\beta$, then the expected wind energy delivered (cleared) is at least $\beta^{*}$ of the total demand, i.e., $\sum_{i \in \mathcal{I}_{r}} \sum_{t=1}^{T} \mathbb{E}\left(\hat{p}_{w_{i}}^{t}\right) \geq$ $\beta^{*} \sum_{i \in \mathcal{I}} \sum_{t=1}^{T} D_{i}^{t}$.

Proof First, as $p_{w_{i}}^{t *}$ are components of an optimal solution to (11), we have $\sum_{i \in \mathcal{I}_{r}} \sum_{t=1}^{T} p_{w_{i}}^{t *} \geq \beta \sum_{i \in \mathcal{I}} \sum_{t=1}^{T} D_{i}^{t}$ immediately due to (11e). Second, (11f) implies that $\mathbb{P}\left\{\hat{p}_{w_{i}}^{t} \geq p_{w_{i}}^{t *}, \forall t \in \mathcal{T}, \forall i \in \mathcal{I}_{r}\right\} \geq 1-\alpha$. Further as $\hat{p}_{w_{i}}^{t} \geq 0$, we have 
$\mathbb{E}\left(\hat{p}_{w_{i}}^{t}\right) \geq(1-\alpha) p_{w_{i}}^{t *}$. We then have

$$
\mathbb{E}\left(\sum_{i \in \mathcal{I}_{r}} \sum_{t=1}^{T} \hat{p}_{w_{i}}^{t}\right) \geq(1-\alpha) \sum_{i \in \mathcal{I}_{r}} \sum_{t=1}^{T} p_{w_{i}}^{t *} \geq \beta(1-\alpha) \sum_{i \in \mathcal{I}} \sum_{t=1}^{T} D_{i}^{t} .
$$

The conclusion that $\mathbb{E}\left(\sum_{i \in \mathcal{I}_{r}} \sum_{t=1}^{T} \hat{p}_{w_{i}}^{t}\right) \geq \beta^{*} \sum_{i \in \mathcal{I}} \sum_{t=1}^{T} D_{i}^{t}$ follows.

Theorem 31 provides a viable method to select $\beta$ for (11) given a static wind power distribution. However, by Remark 3.03, wind power varies over time, which leads to changes in wind power profiles over time.

Corollary 32 Given an RPS mandate $\beta^{*}$ and $T^{\prime}$ days with different $\beta$ values $\left(\beta_{1}, \ldots, \beta_{T^{\prime}}\right)$, let $p_{w_{i}}^{t *}\left(\beta_{1}\right), \ldots, p_{w_{i}}^{t *}\left(\beta_{T^{\prime}}\right)$ be the corresponding optimal solutions of (11). If $\sum_{\tau=1}^{T^{\prime}} \beta_{\tau} / T^{\prime} \geq \beta^{*} /(1-\alpha)$ and the dispatch of wind power $\hat{p}_{w_{i}}^{t}\left(\beta_{\tau}\right)=$ $\min \left\{p_{w_{i}}^{t *}\left(\beta_{\tau}\right), \tilde{p}_{w_{i}}^{t}\right\}, \tau=1, \ldots, T^{\prime}$ is feasible, then the expected wind power delivered is at least $\beta^{*}$ of the total demand over $T^{\prime}$ days, i.e., $\sum_{\tau=1}^{T^{\prime}} \sum_{i \in \mathcal{I}_{r}} \sum_{t=1}^{T} \mathbb{E}\left(\hat{p}_{w_{i}}^{t}\left(\beta_{\tau}\right)\right) \geq \beta^{*} T^{\prime} \sum_{i \in \mathcal{I}} \sum_{t=1}^{T} D_{i}^{t}$.

\section{Solution Methods}

The main difficulty in solving (11) is the computation of the chance constraint (11f). Using SAA, the chance constraint is linearized via new binary variables and big- $M$ constraints, resulting in a hard-to-solve large-scale mixed-integer linear programs. In this paper, we develop a variant of PSAA to approximate chance constraint (11f) by employing a piecewise linear approximation of the cumulative distribution function of $\xi_{1}$. In contrast to SAA, approximate PSAA only introduces new continuous variables and constraints and is thus computationally tractable and scalable to practical size systems.

4.1 Sample average approximation of chance constraint

The chance constraint (11f), repeated as follows for clarity,

$$
\mathbb{P}\left\{p_{w_{i}}^{t} \leq \tilde{p}_{w_{i}}^{t}, \forall t \in \mathcal{T}, \forall i \in \mathcal{I}_{r}\right\} \geq 1-\alpha,
$$

can be reformulated using SAA as:

$$
\begin{aligned}
& p_{w_{i}}^{t}-z_{k} M_{i r}^{k} \leq \tilde{p}_{w_{i}}^{t, k} \forall t \in \mathcal{T}, \forall i \in \mathcal{I}_{r}, k=1, \ldots, N \\
& \frac{\sum_{k=1}^{N} z_{k}}{N} \leq \alpha, z_{k} \in\{0,1\}
\end{aligned}
$$

where the parameter $M_{i r}^{k}$ is chosen to be sufficiently large such that constraints (12) are satisfied when $z_{k}=1$ and $\tilde{p}_{w_{i}}^{t, k}, k=1, \ldots, N$, denote independent scenarios of $\tilde{p}_{w_{i}}^{t}$. It is well known that the particular value of $M_{i r}^{k}$ plays an important role in computational tractability. Typically, as $M_{i r}^{k}$ increases, the linear programming relaxation weakens and fractionality of the resulting MILP increases (and the number of branch-and-bound nodes that must be explored increases). In computational experiments presented in $\S 5, M_{i r}^{k}$ is set to the $\alpha$-quantile of wind power output minus $\tilde{p}_{w_{i}}^{t, k}$. 
4.2 Partial Sample Average Approximation

PSAA leverages the same idea as SAA in that the true distribution of random variables is replaced by an empirical distribution. However, PSAA approximates the distribution of only a subset of random variables rather than all the random variables. Cheng et al. [9] proposed the general idea of PSAA but did not provide guidance on how to partition the random variables into sampled and not-sampled sets. In this paper, we apply SAA to approximate the distribution of all but a single random variable. We then provide guidance and intuition on how to select the single un-sampled random variable and present a piecewise linearization scheme to embed an approximation of the cumulative distribution function of the unsampled random variable directly into the PSAA formulation. Before presenting our method for solving (11), we state our main assumption.

Assumption $\mathcal{A}_{1}$ : The random vector $\left(\tilde{p}_{w_{i}}^{t}\right)_{t \in \mathcal{T} i \in \mathcal{I}_{r}}$ is normally distributed with mean $\mu$ and covariance matrix $\Sigma$.

Remark 4.21 It is widely assumed that wind power production is normally distributed (see e.g., [2, 36, 37]). On the other hand, even if they are not, our proposed approximation method is still applicable provided that: 1) random vector $\left(\tilde{p}_{w_{i}}^{t}\right)_{t \in \mathcal{T} i \in \mathcal{I}_{r}}$ can be represented by a linear combination of random variables of arbitrary distributions and 2) at least one of which is independent of the others and possesses a distribution with an efficiently computable inverse cumulative distribution function.

Under assumption $\mathcal{A}_{1}$, we can reformulate the random vector $\left(\tilde{p}_{w_{i}}^{t}\right)_{t \in \mathcal{T} i \in \mathcal{I}_{r}}$ as follows:

$$
\left(\tilde{p}_{w_{i}}^{t}\right)_{t \in \mathcal{T} i \in \mathcal{I}_{r}}=V \xi+\mu
$$

where $V=\bar{V}(\Lambda)^{\frac{1}{2}}$, and $\Sigma=\bar{V} \Lambda(\bar{V})^{T}$ is the eigen-decomposition of $\Sigma$. Without loss of generality, we assume that the elements of the diagonal of $\Lambda$ are in nonincreasing order. Let $\xi \in \mathbb{R}^{T I_{r}}$ denote a standard normal vector whose components are independent. Analogous to the idea of principal component analysis, we select the random variable with the biggest eigenvalue (i.e., variance) to be the one not sampled $\left(\xi_{1}\right)$. The main motivation for doing so is that the error of SAA is an increasing function of the variance of the estimator. Thus, from an error perspective it is preferable to select $\xi_{2}, \ldots, \xi_{T I_{r}}$, the set of sampled random variables, to be those with the smaller eigenvalues.

With $\xi_{1}$ not sampled, we reconsider constraint (11f). Given (14), constraint (11f) can be reformulated as follows:

$$
\begin{aligned}
p_{w_{i}}^{t} \leq & \tilde{p}_{w_{i}}^{t}=\mathbf{e}_{(t-1) I_{r}+i}^{T}(V \xi+\mu) \\
= & V_{(t-1) I_{r}+i, 1} \xi_{1}+\sum_{l=2}^{T I_{r}} V_{(t-1) I_{r}+i, l} \xi_{l}+\mu_{(t-1) I_{r}+i}, \\
& \quad \forall t \in \mathcal{T} \text { and } \forall i \in \mathcal{I}_{r}
\end{aligned}
$$

where $\mathbf{e}_{l} \in \mathbb{R}^{T I_{r}}, t \in \mathcal{T}$ is a vector where its $l$-th element is one and others are zero, and $V_{(t-1) I_{r}+i, l}$ is the $\left((t-1) I_{r}+i, l\right)$-th entry of the matrix $V$. 
Given assumption $\mathcal{A}_{1}$, the approximate PSAA reformulation of chance constraints (11f) is as follows:

$$
\sum_{k=1}^{N} \frac{\mathbb{P}\left\{p_{w_{i}}^{t}-H_{i}^{t}(k) \leq V_{(t-1) I_{r}+i, 1} \xi_{1}, \forall t \in \mathcal{T} ; i \in \mathcal{I}_{r}\right\}}{N} \geq 1-\alpha
$$

where

$$
H_{i}^{t}(k)=\sum_{l=2}^{T I_{r}} V_{(t-1) I_{r}+i, l} \xi_{l}^{k}+\mu_{(t-1) I_{r}+i}
$$

and $\xi_{l}^{k}, k=1, \ldots, N$ are independent samples of $\xi_{l}$. It is easy to see that $H_{i}^{t}(k)$ is constant. Leveraging the SAA approximation scheme by sampling random variables $\left(\xi_{2}, \ldots, \xi_{T I_{r}}\right)$, constraint (15) may still be computationally intractable. However, constraint (15) can be further approximated using a piecewise linear approximation approach to obtain a set of linearized constraints as follows. There are three cases related to the value of $V_{(t-1) I_{r}+i, 1}$ being positive, negative and zero, which we denote by $\mathcal{I}^{+}, \mathcal{I}^{-}$and $\mathcal{I}^{0}$ respectively. More precisely,

$$
\begin{aligned}
& \xi_{1} \geq \frac{p_{w_{i}}^{t}-H_{i}^{t}(k)}{V_{(t-1) I_{r}+i, 1}} \text { if } V_{(t-1) I_{r}+i, 1}>0, \\
& \xi_{1} \leq \frac{p_{w_{i}}^{t}-H_{i}^{t}(k)}{V_{(t-1) I_{r}+i, 1}} \quad \text { if } V_{(t-1) I_{r}+i, 1}<0 \text {, } \\
& 0 \geq p_{w_{i}}^{t}-H_{i}^{t}(k) \text { if } V_{(t-1) I_{r}+i, 1}=0 \text {. }
\end{aligned}
$$

Note that the three inequalities above correspond to conditions $(t, i) \in \mathcal{I}^{+},(t, i) \in$ $\mathcal{I}^{-}$, and $(t, i) \in \mathcal{I}^{0}$, respectively.

As $\xi_{1}$ is a standard normal random variable, constraint (15) has a deterministic formulation as follows:

$$
\begin{aligned}
& \sum_{k=1}^{N} \frac{\Phi\left(U^{k}\right)-\Phi\left(L^{k}\right)}{N} \mathbb{I}\left\{p_{w_{i}}^{t}-H_{i}^{t}(k) \leq 0,(t, i) \in \mathcal{I}^{0}\right\} \geq 1-\alpha, \\
& U^{k}=\min _{(t, i) \in \mathcal{I}^{-}} \frac{p_{w_{i}}^{t}-H_{i}^{t}(k)}{V_{t(-1) I_{r}+i, 1}}, \quad \forall k=1, \ldots, N \\
& L^{k}=\max _{(t, i) \in \mathcal{I}^{+}} \frac{p_{w_{i}}^{t}-H_{i}^{t}(k)}{V_{(t-1) I_{r}+i, 1}}, \forall k=1, \ldots, N
\end{aligned}
$$

where $\Phi(\cdot)$ is the cumulative distribution function (CDF) of the standard normal distribution. Because of the indicator function $\mathbb{I}(\cdot)$, the first constraint of the reformulation is nonconvex. However, due to the observation that $\mathbb{I}\left\{p_{w_{i}}^{t}-H_{i}^{t}(k) \leq\right.$ $\left.0,(t, i) \in \mathcal{I}^{0}\right\}$ usually takes value 1 when $\alpha$ is close to zero (e.g., 0.05 ), we can obtain a natural (conservative) approximation of constraint (15) as follows:

$$
\begin{aligned}
& \sum_{k=1}^{N} \frac{\Phi\left(U^{k}\right)-\Phi\left(L^{k}\right)}{N} \geq 1-\alpha \\
& p_{w_{i}}^{t}-H_{i}^{t}(k) \leq 0,(t, i) \in \mathcal{I}^{0}, \forall k=1, \ldots, N \\
& U^{k}=\min _{(t, i) \in \mathcal{I}^{-}} \frac{p_{w_{i}}^{t}-H_{i}^{t}(k)}{V_{(t-1) I_{r}+i, 1}}, \forall k=1, \ldots, N \\
& L^{k}=\max _{(t, i) \in \mathcal{I}^{+}} \frac{p_{w_{i}}^{t}-H_{i}^{t}(k)}{V_{(t-1) I_{r}+i, 1}}, \forall k=1, \ldots, N
\end{aligned}
$$




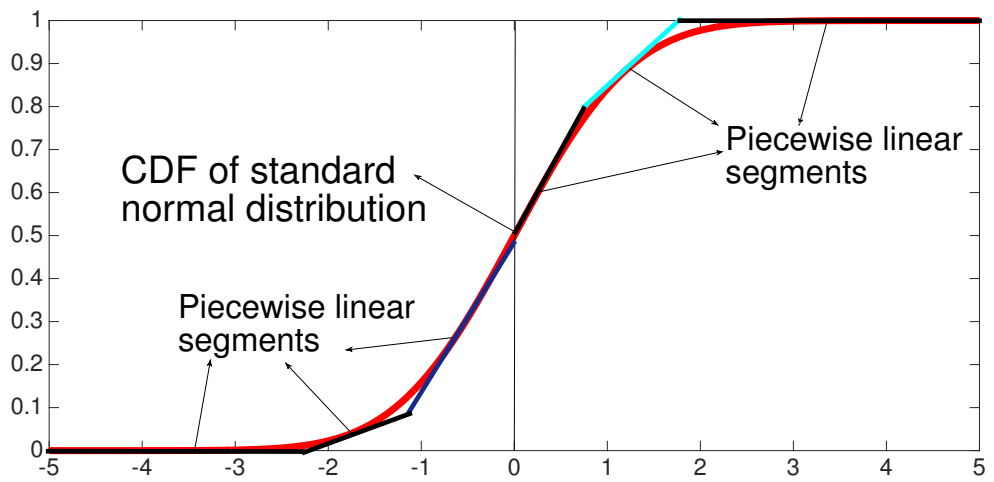

Fig. 1: Piecewise linear approximation of the CDF of a standard normal random variable.

As $\alpha$ is usually close to zero (e.g., 0.05$), \Phi\left(U^{k}\right)$ is usually greater than 0.5 , thus $U^{k}>0$, while $\Phi\left(L^{k}\right)$ is usually less than 0.5 , thus $L^{k}<0$. Figure 1 illustrates $\Phi(z)$ and how it can be upper bounded by a piecewise linear concave function which is tangent to the actual function at a finite number of points when $z \geq 0$ while it can be lower bounded by a piecewise linear convex function when $z \leq 0$. To this end, we approximate $\Phi\left(U^{k}\right)$ with a piecewise linear concave function

$$
\Phi\left(U^{k}\right) \approx \min _{s \in\{1, \ldots, S\}} \hat{a}_{s} U^{k}+\hat{b}_{s}
$$

while $\Phi\left(L^{k}\right)$ is approximated by a piecewise linear convex function

$$
\Phi\left(L^{k}\right) \approx \max _{s \in\{1, \ldots, S\}} \bar{a}_{s} L^{k}+\bar{b}_{s}
$$

where $s \in\{1, \ldots, S\}$ indexes each of the $S$ linear segments on either side of zero, with corresponding slopes and intercepts $\hat{a}_{s}, \hat{b}_{s}$ for $U^{k}$, and $\bar{a}_{s}, \bar{b}_{s}$ for $L^{k}$. In our experiments, we choose 25 tangent points on the interval $[-3,3]$ for the tangent approximation. It is easy to find that the more tangent points we choose, the more accurate the approximation will be, whereas at the same time this will increase the size of problem.

With the piecewise linear approximation of $\Phi\left(U^{k}\right)$ and $\Phi\left(L^{k}\right)$ respectively, we obtain the following approximation of the PSAA problem by introducing auxiliary 
variables $\bar{U}^{k}$ and $\bar{L}^{k}$ to represent $\Phi\left(U^{k}\right)$ and $\Phi\left(L^{k}\right)$ respectively:

$$
\begin{array}{ll}
\min & \sum_{g \in \mathcal{G}} \sum_{t \in \mathcal{T}} C_{g}^{p}\left(p_{g}^{t}\right) \\
\text { s.t. } & (10 \mathrm{c})-(10 \mathrm{f}),(11 \mathrm{a})-(11 \mathrm{e}) \\
& \sum_{k=1}^{N} \frac{\left(\bar{U}^{k}-\bar{L}^{k}\right)}{N} \geq 1-\alpha \\
& U^{k} \leq \frac{p_{w_{i}}^{t}-H_{i}^{t}(k)}{V_{(t-1) I_{r}+i, 1}},(t, i) \in \mathcal{I}^{-}, \forall k=1, \ldots, N \\
& L^{k} \geq \frac{p_{w_{i}}^{t}-H_{i}^{t}(k)}{V_{(t-1) I_{r}+i, 1}},(t, i) \in \mathcal{I}^{+}, \forall k=1, \ldots, N \\
& p_{w_{i}}^{t}-H_{i}^{t}(k) \leq 0,(t, i) \in \mathcal{I}^{0}, \forall k=1, \ldots, N \\
& \bar{U}^{k} \leq \hat{a}_{s} U^{k}+\hat{b}_{s}, \forall s=1, \ldots, S, k=1, \ldots, N \\
& \bar{L}^{k} \geq \bar{a}_{s} L^{k}+\bar{b}_{s}, \forall s=1, \ldots, S, k=1, \ldots, N \\
& \bar{U}^{k} \geq \bar{L}^{k} \geq 0, \forall k=1, \ldots, N
\end{array}
$$

This model employs a convex quadratic objective function and linear constraints.

\section{Computational Experiments}

We now conduct numerical experiments to compare PSAA to three popular approximations for chance constrained problems: SAA, Bonferroni approximation and the scenario approach (SA) using a modified IEEE 24-bus system [40] and the WECC 240-bus system (representing the US Western Interconnection [25]). We added 3 wind farms, at buses 7, 13, and 15, in the IEEE 24-bus system and 10 wind farms, at buses $22,23,42,44,54,64,77,129,174$, and 240, in the WECC 240-bus system. We assume storage capacity is identical at all buses with wind farms. All optimization models are implemented in Matlab and solved using Gurobi 6.0.4 [15]. We impose a 3600 second time limit and an optimality gap of $0.1 \%$ as the stopping criteria for all runs. All experiments were performed on a Linux workstation with an Intel Quad Core $2.2 \mathrm{GHz}$ processor and $16 \mathrm{~GB}$ memory.

The IEEE 24-bus system consists of 32 conventional generators, 34 transmission lines, 17 loads, and three wind farms. Data for the three wind farms are based on NREL's Western Wind Dataset [17]. We selected two geographically close wind farms located in Wyoming (site \#15414 (41.48N,105.14W) and site \#16238 $(41.61 N, 105.11 W))$ and a single site in California ( site \#3560 $(35.08 N, 118.41 W)$ ). We model the uncertain wind speed as a random field over time, and construct a representation using the $L^{2}$-optimal Karhunen-Loeve (KL) expansion [14]. Using the KL representations for the wind speed we generated samples that are consistent with historical data and also with the magnitude of errors for day-ahead wind forecasts. These samples were converted to power by interpolation using the wind turbine's rated power curve. A detailed description of this approach is presented in [29]. We then use these samples to estimate the mean and covariance. We set the total installed wind capacity to be $1500 \mathrm{MW}$ in order to meet high RPS requirements (33\%). The remaining parameters are set as follows: $\alpha=0.05$, 


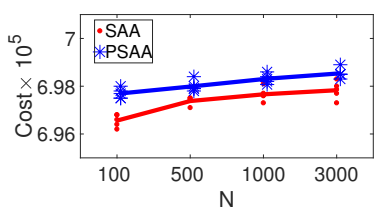

(a) Solution Cost

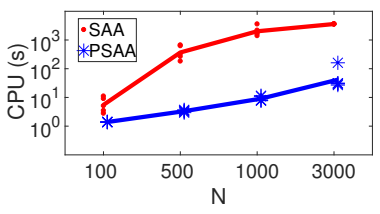

(b) Runtime

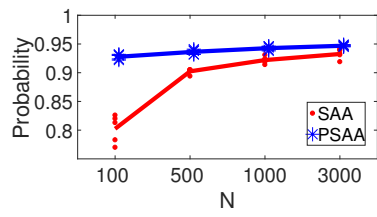

(c) Robustness

Fig. 2: Comparison of SAA and PSAA for the IEEE 24-bus system

$\beta=33 \%$, and $T=24$ hours. The storage capacity is set to be $1 / 5$ of installed hourly wind capacity (i.e., $300 \mathrm{MWh}$ ) while the initial storage level is set to $1 / 4$ of storage capacity. For simplicity, we assume that charge and discharge rates in one hour are identical to capacity (i.e., $300 \mathrm{MWh}$ ). We consider four settings for the number of scenarios used: $N \in\{100,500,1000,3000\}$. In contrast to Bonferroni approximation, SAA, PSAA and SA solutions are functions of the realized input scenarios. Thus, to compare the performance of these approaches, five sets of scenarios for each $N$ are considered. We illustrate the performance of both SAA and PSAA in Figure 2, which shows how solution cost, CPU time, and empirical probability of the solution change with increasing number of samples. Here, empirical probability of solution is defined as the proportion of $N_{p}$ samples satisfying constraint $p_{w_{i}}^{t} \leq \tilde{p}_{w_{i}}^{t}, \forall t \in \mathcal{T}, \forall i \in \mathcal{I}_{r}$ given solution $p_{w_{i}}^{t}$. For our analysis, we set $N_{p}=100,000$. These results are summarized in Table 1.

\subsection{Comparison of SAA and PSAA on the 24-Bus System.}

Figure $2 \mathrm{a}$ and Table 1 indicate there is no significant difference between the SAA and PSAA approaches in terms of solution cost. PSAA solution cost is slightly higher than SAA solution cost when $N \leq 1000$ because PSAA solutions have higher empirical probability. For $N=3000$, SAA reaches the stopping criteria of 3600 seconds and thus the solutions we collect are incumbent solutions (best feasible solutions so far) rather than optimal solutions. Incumbent solutions are more conservative than optimal solutions, which may explain the observed differences in solution cost.

Figure $2 \mathrm{~b}$ compares the runtime performance of SAA and PSAA. The results show that PSAA yields approximately a two orders of magnitude reduction in computation time relative to SAA for $N \geq 500$. In particular, PSAA only requires 56 seconds for the $N=3000$ case, whereas the SAA approach failed to establish an optimal solution after one hour.

The quality of the solutions obtained by SAA and PSAA in terms of empirical probability is presented in Figure 2c. PSAA solutions provide improved empirical probabilities, and are closer to the target satisfaction threshold of $1-\alpha=0.95$. For example, when $N=100$, the empirical probability of the SAA solution is less than 0.81 , whereas it is 0.928 for the PSAA approach. Overall, the PSAA requires far fewer scenarios to obtain a high-quality solution compared to the SAA approach.

When compared with Bonferroni approximation and scenario approach, PSAA yields a less conservative (lower cost) solution at the expense of a slightly higher 
Table 1: Comparisons of SAA and PSAAA on average statistics on 5 instances.

\begin{tabular}{|c||c|c|c|c|c|c|}
\hline \multicolumn{1}{|c||}{$\alpha=0.05$} & \multicolumn{3}{c|}{ PSAA } \\
\hline $\mathrm{N}$ & Cost(\$10K) & CPU(s) & Prob & Cost $(\$ 10 K)$ & CPU(s) & Prob \\
\hline 100 & 6.966 & 6.3 & 0.802 & 6.977 & 1.4 & 0.928 \\
500 & 6.974 & 415.0 & 0.902 & 6.980 & 3.4 & 0.936 \\
1000 & 6.977 & 2127.8 & 0.922 & 6.983 & 9.2 & 0.943 \\
3000 & 6.982 & 3600 & 0.933 & 6.985 & 55.9 & 0.947 \\
\hline$\alpha=0.05$ & \multicolumn{3}{|c|}{ SA } & \multicolumn{3}{c|}{ Bonferroni } \\
\hline $\mathrm{N}$ & Cost $(\$ 10 \mathrm{~K})$ & $\mathrm{CPU}(\mathrm{s})$ & Prob & Cost $(\$ 10 \mathrm{~K})$ & CPU(s) & Prob \\
\hline 100 & 6.996 & 0.7 & 0.877 & 7.081 & 0.7 & 0.987 \\
500 & 7.057 & 0.8 & 0.969 & 7.081 & 0.7 & 0.987 \\
1000 & 7.130 & 0.9 & 0.984 & 7.081 & 0.7 & 0.987 \\
3000 & 7.179 & 1.0 & 0.992 & 7.081 & 0.7 & 0.987 \\
\hline
\end{tabular}

runtime. For instance, when $N=3000$, PSAA takes 56 seconds to solve the problem while both Bonferroni approximation and scenario approach solved within 1 second. But their optimal solutions are $\$ 960$ and $\$ 1940$ higher than the PSAA solution, respectively. From these numerical results, it can be concluded that PSAA is an efficient method for solving this class of chance-constrained optimization problems, providing a nice tradeoff between computational tractability and rapid convergence to high-quality solutions.

\subsection{Sensitivity to Storage Capacity}

We now test the sensitivity of solution cost to variations in installed wind and storage capacities. We tested combinations of 6 storage capacities $(0,200,400,600,800$, and $1000 \mathrm{MWh})$ and 4 installed wind capacities (1350, 1500, 1650, $1800 \mathrm{MW})$. The results are summarized in Figure 3. First, as expected, we observe that cost decreases as storage capacity and/or installed wind capacity increases. Second, we observe that only marginal improvements in costs are obtained once installed wind capacity or storage capacity exceed a certain threshold. For instance, when installed wind capacity is $1500 \mathrm{MW}$, increasing storage capacity from 0 to 200 results in cost reductions of more than $\$ 2000$ while an increased storage capacity, from 200 to 400 , only reduces the cost by $\$ 500$. Moreover, when installed wind capacities are sufficiently large, increasing storage capacities yield no further cost reductions.

\subsection{Comparison of SAA and PSAA on the 240-Bus System}

The WECC 240-bus test system consists of 85 conventional generators, 348 transmission lines, 142 loads, and 10 wind farms. Wind power uncertainty is modeled similarly to the approach employed in Section 5.1. We set the installed wind capacity to $510 \mathrm{MW}$. The remaining parameters are identical to those used in our analysis of the 24-bus system. Because our focus here is to demonstrate the tractability of PSAA, we selected a large number of scenario samples to test $(N=1000,3000$, and 5000). Additionally, timing results for these large instances using a linear cost function are also presented. The results in Table 2 demonstrate the tractability of PSAA and the intractability of SAA, which failed to solve any of the larger instances to optimality within the given time limit. Although expected, increased computational times are observed for these larger instances. However, the largest 


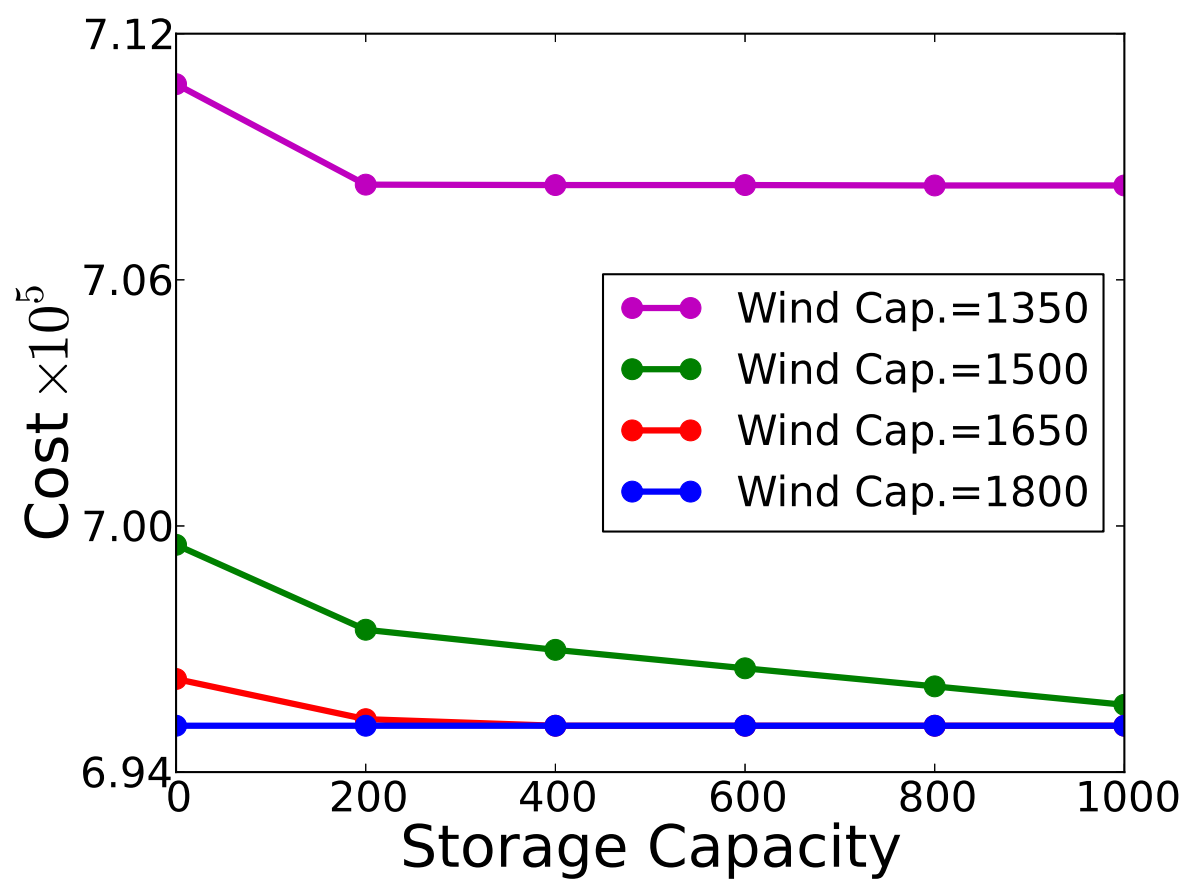

Fig. 3: Solution cost on 24-bus test system under varying wind and storage capacities.

instance with 5000 scenarios can still be solved using PSAA within 700 seconds using a quadratic objective function. When a linear approximation of the cost function is employed, the runtime drops to less than 60 seconds. When comparing PSAA with Bonferroni approximation and scenario approach, we can draw similar conclusions as the 24-bus system. Although PSAA requires a longer runtime, both Bonferroni approximation and scenario approach find overly conservative (highcost) solutions. For instance, when $N=5000$ and the quadratic cost function is employed, the costs of the solutions provided by Bonferroni approximation and scenarios approach are $66.1 \%$ and $92.6 \%$ higher, respectively, than the one of the PSAA solution.

\subsection{Comparison of SAA and PSAA on Large-Size Power System}

In this subsection, we compare the performance of SAA and PSAA approaches on a synthetic large-scale power system. Following the same construction procedure in [11], we quadruplicate the WECC 240-bus system to construct a 960-bus system with 1422 transmission line, 340 conventional generators, 568 loads, and 40 wind farms. The remaining parameters are identical to those used in our analysis of the WECC 240-bus system. Numerical results are shown in Table (3). Again, the results in Table 3 demonstrate the intractability of SAA, which failed to solve any of the larger instances to optimality within the given time limit. Although PSAA also fails to solve the quadratic objective instance to optimality when $N=5000$, it took only 200 seconds (or less) to solve all the large-scale instances with a linear 
Table 2: Comparison of SAA and PSAA for WECC-240. "-" indicates that no solution found in one hour.

\begin{tabular}{|c|c|c|c|c|c|c|}
\hline & \multicolumn{6}{|c|}{ Quadratic cost function } \\
\hline$\alpha=0.05$ & \multicolumn{3}{|c|}{ SAA } & \multicolumn{3}{|c|}{ PSAA } \\
\hline $\mathrm{N}$ & $\operatorname{Cost}\left(10^{6} \$\right)$ & $\mathrm{CPU}(\mathrm{s})$ & Prob & $\operatorname{Cost}\left(10^{6} \$\right)$ & $\mathrm{CPU}(\mathrm{s})$ & Prob \\
\hline 1000 & 2.059 & 3600 & 0.945 & 1.850 & 35.5 & 0.930 \\
\hline 3000 & - & - & - & 1.953 & 289.2 & 0.945 \\
\hline 5000 & - & - & - & 2.064 & 681.5 & 0.947 \\
\hline$\alpha=0.05$ & \multicolumn{3}{|c|}{$\mathrm{SA}$} & \multicolumn{3}{|c|}{ Bonferroni } \\
\hline $\mathrm{N}$ & $\operatorname{Cost}\left(10^{6} \$\right)$ & $\mathrm{CPU}(\mathrm{s})$ & Prob & $\operatorname{Cost}\left(10^{6} \$\right)$ & $\mathrm{CPU}(\mathrm{s})$ & Prob \\
\hline 1000 & 2.433 & 1.4 & 0.958 & 3.429 & 0.8 & 0.991 \\
\hline 3000 & 3.259 & 2.3 & 0.983 & 3.429 & 0.8 & 0.991 \\
\hline 5000 & 3.975 & 3.1 & 0.991 & 3.429 & 0.8 & 0.991 \\
\hline & \multicolumn{6}{|c|}{ Linear cost function } \\
\hline$\alpha=0.05$ & \multicolumn{3}{|c|}{ SAA } & \multicolumn{3}{|c|}{$\overline{\text { PSAA }}$} \\
\hline $\mathrm{N}$ & $\operatorname{Cost}\left(10^{6} \$\right)$ & $\mathrm{CPU}(\mathrm{s})$ & Prob & $\operatorname{Cost}\left(10^{6} \$\right)$ & $\mathrm{CPU}(\mathrm{s})$ & Prob \\
\hline 1000 & 1.951 & 3600 & 0.930 & 1.899 & 12.0 & 0.928 \\
\hline 3000 & 2.502 & 3600 & 0.963 & 1.996 & 36.4 & 0.945 \\
\hline 5000 & 3.312 & 3600 & 0.984 & 2.107 & 55.3 & 0.947 \\
\hline$\alpha=0.05$ & \multicolumn{3}{|c|}{ SA } & \multicolumn{3}{|c|}{ Bonferroni } \\
\hline $\mathrm{N}$ & $\operatorname{Cost}\left(10^{6} \$\right)$ & $\mathrm{CPU}(\mathrm{s})$ & Prob & $\operatorname{Cost}\left(10^{6} \$\right)$ & $\mathrm{CPU}(\mathrm{s})$ & Prob \\
\hline 1000 & 2.483 & 0.9 & 0.957 & 3.513 & 0.7 & 0.992 \\
\hline 3000 & 3.351 & 1.2 & 0.983 & 3.513 & 0.7 & 0.992 \\
\hline 5000 & 4.067 & 1.4 & 0.991 & 3.513 & 0.7 & 0.992 \\
\hline
\end{tabular}

objective function. We also note that the empirical probability of PSAA solution is not close to the target threshold of $1-\alpha=0.95$ even when $N=5000$. However, it is worth noting that the dimension of the random variables involved is 960 . Given the high dimensionality of the random variables, even PSAA would require a much larger number of scenarios to obtain a high-quality solution.

Table 3: Comparison of SAA and PSAA for 960-bus system. "-" indicates that no solution found in one hour.

\begin{tabular}{|c||c|c|c|c|c|c|}
\hline \multicolumn{1}{|c||}{} & \multicolumn{5}{c|}{ Quadratic cost function } \\
\hline$\alpha=0.05$ & \multicolumn{3}{c|}{ SAA } & \multicolumn{3}{c|}{ PSAA } \\
\hline $\mathrm{N}$ & Cost $\left(10^{7} \$\right)$ & $\mathrm{CPU}(\mathrm{s})$ & Prob & Cost $\left(10^{7} \$\right)$ & $\mathrm{CPU}(\mathrm{s})$ & Prob \\
\hline 1000 & - & - & - & 4.431 & 1361.7 & 0.892 \\
3000 & - & - & - & 4.496 & 3423.5 & 0.926 \\
5000 & - & - & - & - & - & - \\
\hline \multicolumn{7}{|c|}{ SAA } \\
\hline$\alpha=0.05$ & \multicolumn{7}{|c|}{ Linear cost function } \\
\hline $\mathrm{N}$ & Cost $\left(10^{7} \$\right)$ & $\mathrm{CPU}(\mathrm{s})$ & Prob & Cost $\left(10^{7} \$\right)$ & $\mathrm{CPU}(\mathrm{s})$ & Prob \\
\hline 1000 & - & - & - & 4.852 & 22.3 & 0.887 \\
3000 & - & - & - & 4.953 & 72.8 & 0.925 \\
5000 & - & - & - & 5.067 & 200.8 & 0.932 \\
\hline
\end{tabular}

\section{Conclusion}

We presented a new formulation for the chance-constrained economic dispatch (ED) problem which integrates renewable energy and energy storage. The chanceconstrained ED problem is among the most computationally challenging electricity grid operations problems and an important component of an integrated UC/ED operations planning process given renewables and storage. Currently, state-of-theart approaches are limited to small-scale problems, considering only a small num- 
ber of renewable resources and/or limited to a small number of scenarios. We also proposed a novel solution method based on partial sample average approximation (PSAA) to enable tractable solution of large-scale instances.

Our formulation uses chance constraints to ensure that the scheduled wind energy is ultimately dispatchable and the portion of demand met with wind resources is at or above a specified ratio with high probability. This formulation directly mirrors the structure of RPS mandates. Standard sample average approximation (SAA) approaches lead to mixed-integer problems which are generally hard to solve. Here, we adopt PSAA to quickly obtain high-quality solutions. PSAA results in a convex program and is thus computationally tractable for large instances. The effectiveness of PSAA is illustrated by experiments on the IEEE 24-bus and WECC 240-bus test systems. In our model, we follow convention and assume that wind power is normally distributed. A natural line of further research lies in generalizing our PSAA approach to other distributions (e.g., Weibull distribution). Applying our approach to unit commitment is another promising direction.

\section{Appendix A}

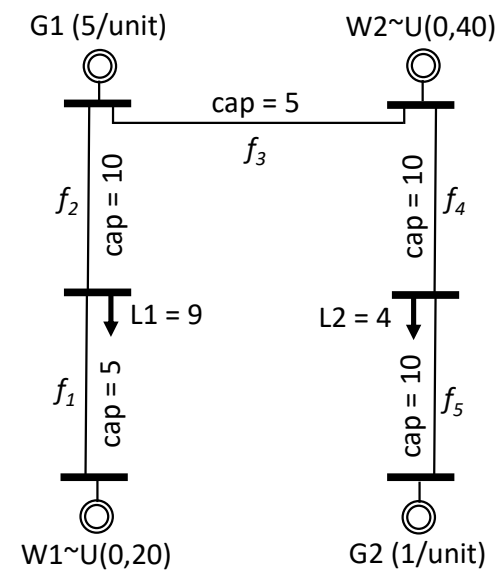

Fig. 4: Simplified power grid.

We consider a simplified 6-bus system illustrated in Figure 4. In this system, there are two conventional generators with capacity 10 and 20 (with cost 5 and 1 per unit, respectively). There are two wind farms with independent uniformly distributed capacity on the intervals $[0,20]$ and $[0,40]$. There are two load buses with loads 9 and 4 units, respectively, and five transmission lines with capacities of 5 and 10 units. We choose a confidence parameter $\alpha=0.19$ for constraint (11f) 
(chance constraint) and $\beta=50 \%$ for constraint (11e). Accordingly, we have the following optimization problem.

$$
\begin{array}{cl}
\min _{\boldsymbol{f}, \boldsymbol{p}} & 5 p_{1}+p_{2} \\
\text { s.t. } & f_{1}-p_{w_{1}}=0, f_{1}-f_{2}=9, \\
& f_{2}-f_{3}+p_{1}=0, f_{3}-f_{4}+p_{w_{2}}=0 \\
& f_{4}-f_{5}=4, f_{5}+p_{2}=0, \\
& \left|f_{i}\right| \leq 5, i=1,3,\left|f_{i}\right| \leq 10, i=2,4,5 \\
& 0 \leq p_{1} \leq 10,0 \leq p_{2} \leq 20, p_{w_{1}}, p_{w_{2}} \geq 0 \\
& p_{w_{1}}+p_{w_{2}} \geq 6.5, \\
& \mathbb{P}\left\{p_{w_{1}} \leq \tilde{p}_{w_{1}}, p_{w_{2}} \leq \tilde{p}_{w_{2}}\right\} \geq 0.81
\end{array}
$$

which is equivalent to the following problem:

$$
\begin{array}{cl}
\min _{\boldsymbol{p}} & 5 p_{1}+p_{2} \\
\text { s.t. } & \left|p_{w_{1}}\right| \leq 5,\left|p_{w_{1}}-9\right| \leq 10,\left|p_{w_{1}}-9+p_{1}\right| \leq 5, \\
& \left|p_{w_{1}}-9+p_{1}+p_{w_{2}}\right| \leq 10 \\
& \left|p_{w_{1}}-13+p_{1}+p_{w_{2}}\right| \leq 10 \\
& p_{w_{1}}-13+p_{1}+p_{w_{2}}+p_{2}=0 \\
& 0 \leq p_{1} \leq 10,0 \leq p_{2} \leq 20, p_{w_{1}}, p_{w_{2}} \geq 0 \\
& p_{w_{1}}+p_{w_{2}} \geq 6.5 \\
& \mathbb{P}\left\{p_{w_{1}} \leq \tilde{p}_{w_{1}}, p_{w_{2}} \leq \tilde{p}_{w_{2}}\right\} \geq 0.81
\end{array}
$$

The optimal solution of problem (22) is $\boldsymbol{p}=\left(p_{1}, p_{2}, p_{w_{1}}, p_{w_{2}}\right)=$ $(2.532,3.968,1.468,5.032)$ with the optimal value 16.628 . Without constraint $(22 \mathrm{~g})$, the optimal solution is $\boldsymbol{p}=(0.2,9,3.8,0)$ with the optimal value 10 . Upon further investigation, we found that constraint $(22 \mathrm{~g})$ is necessary because of transmission constraints. Without transmission limit, it is always the case that the objective function is a non-increasing function of the total penetration of wind energy.

Additionally, we consider three cases: $\boldsymbol{p}_{1}=(4,1.5,0,7.5), \boldsymbol{p}_{2}=(3,3.5,1,5.5)$ and $\boldsymbol{p}_{3}=(2,5,2,4)$. Without constraint $(22 \mathrm{~g})$, all three solutions are feasible solution to problem (22) with cost $21.5,18.5$ and 15 , respectively. Although $\boldsymbol{p}_{3}$ has the smallest cost among them, it does not satisfy constraint (22g). Moreover compared with solution $\boldsymbol{p}_{1}, \boldsymbol{p}_{2}$ has a smaller cost but it also has a lower wind penetration. Thus, we can conclude that (1) constraint $(22 \mathrm{~g})$ is not redundant and (2) maximum dispatch of (free) wind does not necessitate a lower cost for general power systems, due to the transmission limitations.

\section{References}

1. Bertsimas, Dimitris, Eugene Litvinov, Xu Andy Sun, Jinye Zhao, and Tongxin Zheng. "Adaptive robust optimization for the security constrained unit commitment problem." IEEE

Transactions on Power Systems, 28, no. 1 (2013): 52-63. 
2. Bienstock, Daniel, Michael Chertkov, and Sean Harnett. "Chance-constrained optimal power flow: Risk-aware network control under uncertainty." SIAM Review 56.3 (2014): 461495.

3. California Renewables Portfolio Standard (RPS), http://www.cpuc.ca.gov/PUC/energy/ Renewables/.

4. Calafiore, Giuseppe C., and Marco C. Campi. "The scenario approach to robust control design." IEEE Transactions on Automatic Control 51, no. 5 (2006): 742-753.

5. Castillo, Anya, and Dennice F. Gayme. "Grid-scale energy storage applications in renewable energy integration: A survey." Energy Conversion and Management 87 (2014): 885-894.

6. Chandy, K. Mani, Steven H. Low, Ufuk Topcu, and Huan Xu. "A simple optimal power flow model with energy storage." In Decision and Control (CDC), 2010 49th IEEE Conference on, pp. 1051-1057. IEEE, 2010.

7. Charnes, Abraham, William W. Cooper, and Gifford H. Symonds. "Cost horizons and certainty equivalents: an approach to stochastic programming of heating oil." Management Science 4, no. 3 (1958): 235-263.

8. Cheng, Jianqiang, and Abdel Lisser. "A second-order cone programming approach for linear programs with joint probabilistic constraints." Operations Research Letters 40, no. 5 (2012): 325-328.

9. Cheng, Jianqiang, Céline Gicquel, and Abdel Lisser. "A New partial sample average approximation method for chance-constrained problems." http://www.optimization-online. org/DB_FILE/2014/11/4622.pdf.

10. Cheung, K., D. Gade, C. S. Monroy, S. M. Ryan, J. P. Watson, R. J. B. Wets, and D. L. Woodruff. "Toward scalable stochastic unit commitment-part 2: Assessing solver performance." Energy Systems 6(3), pp. 417-438, 2015.

11. Dashti, Hossein, Antonio J. Conejo, Ruiwei Jiang, and Jianhui Wang. "Weekly two-stage robust generation scheduling for hydrothermal power systems." IEEE Transactions on Power Systems 31, no. 6 (2016): 4554-4564.

12. " $20 \%$ Wind Energy by 2030: Increasing wind energy's contribution to the US electricity supply." DOE/GO-102008-2567, US Department of Energy, May. http://www.nrel.gov/ docs/fy08osti/41869.pdf, 2008.

13. Gayme, Dennice, and Ufuk Topcu. "Optimal power flow with large-scale storage integration." IEEE T. Power Systems, 28, no. 2 (2013): 709-717.

14. Ghanem, Roger, and John RedHorse. "Propagation of probabilistic uncertainty in complex physical systems using a stochastic finite element approach." Physica D, 133, no. 1 (1999): 137-144.

15. Gurobi Optimization, Inc. "Gurobi optimizer reference manual." http://www. gurobi.com, 2015.

16. Jiang, Ruiwei, Jianhui Wang, and Yongpei Guan. "Robust unit commitment with wind power and pumped storage hydro." IEEE Transactions on Power Systems, 27, no. 2 (2012): 800-810.

17. Lew, Debra, and R. Piwko. "Western wind and solar integration study." National Renewable Energy Laboratories, Technical Report No. NREL/SR-550-47781 (2010).

18. Lorca, Alvaro, and Xu Andy Sun. "Adaptive robust optimization with dynamic uncertainty sets for multi-period economic dispatch under significant wind." IEEE T. Power Systems, 30.4 (2015): 1702-1713.

19. Luedtke, James, and Shabbir Ahmed. "A sample approximation approach for optimization with probabilistic constraints." SIAM J. Optimization 19.2 (2008): 674-699.

20. Nemirovski, Arkadi, and Alexander Shapiro. "Convex approximations of chanceconstrained programs." SIAM J. Optimization 17.4 (2006): 969-996.

21. NREL: Transmission Grid Integration - Western Wind Dataset, http://www.nrel.gov/ electricity/transmission/wind_integration_dataset.html.

22. Padhy, Narayana Prasad. "Unit commitment-a bibliographical survey." IEEE T. Power Systems, 19, no. 2 (2004): 1196-1205.

23. Pagnoncelli, Bernardo K., Shabbir Ahmed, and Alexander Shapiro. "Sample average approximation method for chance-constrained programming: theory and applications." J. optimization theory and applications 142.2 (2009): 399-416.

24. Prékopa, András. "Probabilistic programming. "Handbooks in operations research and management science 10 (2003): 267-351.

25. Price, James E., and John Goodin. "Reduced network modeling of WECC as a market design prototype." IEEE Power and Energy Society General Meeting, pp. 1-6. IEEE, 2011. 
26. Qiu, Feng, and Jianhui Wang. "Chance-constrained transmission switching with guaranteed wind power utilization." IEEE T. Power Systems, 30, no. 3 (2015): 1270-1278.

27. Rockafellar, R. Tyrrell, and Roger J-B. Wets. "Scenarios and policy aggregation in optimization under uncertainty." Mathematics of operations research 16, no. 1 (1991): 119-147.

28. Jocelyn Durkay (2017) State Renewable Portfolio Standards and Goals. http://www.ncsl.org/research/energy/renewable-portfolio-standards.aspx. Accessed 14 Nov. 2017

29. Safta, Cosmin, Richard L-Y. Chen, Habib N. Najm, Ali Pinar, and Jean-Paul Watson. "Efficient uncertainty quantification in stochastic economic dispatch." IEEE Transactions on Power Systems 32, no. 4 (2017): 2535-2546.

30. Shapiro, Alexander, Darinka Dentcheva, and Andrzej Ruszczyński. "Lectures on stochastic programming: modeling and theory." Vol. 16. SIAM, 2014.

31. Silva-Monroy, Cesar, and Jean-Paul Watson. "Integrating Energy Storage Devices Into Market Management Systems." Proceedings of the IEEE 102, no. 7 (2014): 1084-1093.

32. Song, Yongjia, James R. Luedtke, and Simge Küçükyavuz. "Chance-constrained binary packing problems." INFORMS Journal on Computing 26, no. 4 (2014): 735-747.

33. Su, Han-I., and Abbas El Gamal. "Modeling and analysis of the role of energy storage for renewable integration: Power balancing." IEEE T. Power Systems, 28, no. 4 (2013): 4109-4117.

34. Ummels, Bart C., Madeleine Gibescu, Engbert Pelgrum, Wil L. Kling, and Arno J. Brand. "Impacts of wind power on thermal generation unit commitment and dispatch." IEEE T. Energy Conversion, 22, no. 1 (2007): 44-51.

35. Van Slyke, Richard M., and Roger Wets. "L-shaped linear programs with applications to optimal control and stochastic programming." SIAM J. Applied Mathematics 17, no. 4 (1969): 638-663.

36. Wang, Jianhui, Mohammad Shahidehpour, and Zuyi Li. "Security-constrained unit commitment with volatile wind power generation." IEEE Transactions on Power Systems 23, no. 3 (2008): 1319-1327.

37. Wang, Qianfan, Yongpei Guan, and Jianhui Wang. "A chance-constrained two-stage stochastic program for unit commitment with uncertain wind power output." IEEE T. Power Systems, 27, no. 1 (2012): 206-215.

38. Wood, Allen J., and Bruce F. Wollenberg. Power generation, operation, and control. John Wiley \& Sons, 2012.

39. Wu, Hongyu, Mohammad Shahidehpour, Zuyi Li, and Wei Tian. "Chance-constrained day-ahead scheduling in stochastic power system operation." IEEE T. Power Systems, 29, no. 4 (2014): 1583-1591.

40. Zimmerman, Ray Daniel, Carlos Edmundo Murillo-Sánchez, and Robert John Thomas. "MATPOWER: Steady-state operations, planning, and analysis tools for power systems research and education." Power Systems, IEEE T.26, no. 1 (2011): 12-19.

41. Zhang, Yu, Nikolaos Gatsis, and Georgios B. Giannakis. "Robust Energy Management for Microgrids With High Penetration Renewables." IEEE T. on Sustainable Energy, 4, no. 4 (2013): $944-953$. 